\title{
Stochastic Orderings among Order Statistics and Sample Spacings
}

\author{
BAHA-ELDIN KHALEDI \\ and \\ SubHaSh KOCHAR
}

April 24, 2002

isid/ms/2002/09

Indian Statistical Institute, Delhi Centre

7, SJSS Marg, New Delhi-110 016, India 


\title{
Stochastic Orderings among Order Statistics and Sample Spacings
}

\author{
Baha-Eldin Khaledi \\ Dept. of Statistics, College of Science, \\ Razi University, Kermanshah, Iran \\ e-mail: bkhaledi@hotmail.com \\ Subhash Kochar \\ Indian Statistical Institute, 7, SJS Sansanwal Marg \\ New Delhi-110016, India \\ e-mail : kochar@isid.ac.in
}

April 24, 2002

\begin{abstract}
In this paper we review some of the results obtained recently in the area of stochastic comparisons of order statistics and sample spacings. We consider the cases when the parent observations are identically as well as non-identically distributed. But most of the time we shall be assuming that the observations are independent. The case of independent exponentials with unequal scale parameters is discussed in detail.
\end{abstract}

\section{Introduction}

The simplest and the most common way of comparing two random variables is through their means and variances. It may happen that in some cases the median of $X$ is larger than that of $Y$, while the mean of $X$ is smaller than the mean of $Y$. However, this confusion will not arise if the random variables are stochastically ordered. Similarly, the same may happen if one would like to compare the variability of $X$ with that of $Y$ based only on numerical measures like standard deviation etc. Besides, these characteristics of distributions might not exist in some cases. In most cases one can express various forms of knowledge about the underlying distributions in terms of their survival functions, hazard rate functions, mean residual functions, quantile functions and other suitable functions of probability distributions. These methods are much more informative than those based only on few numerical characteristics of distributions. Comparisons of random variables based on such functions usually establish partial orders among them. We call them as stochastic orders. 
Stochastic models are usually sufficiently complex in various fields of statistics, particularly in reliability theory. Obtaining bounds and approximations for their characteristics is of practical importance. That is, the approximation of a stochastic model either by a simpler model or by a model with simple constituent components might lead to convenient bounds and approximations for some particular and desired characteristics of the model. The study of changes in the properties of a model, as the constituent components vary, is also of great interest. Accordingly, since the stochastic components of models involve random variables, the topic of stochastic orders among random variables plays an important role in these areas.

Order statistics and spacings are of great interest in many areas of statistics and they have received a lot of attention from many researchers. Let $X_{1}, \ldots, X_{n}$ be $n$ random variables. The $i$ th order statistic, the $i$ th smallest of $X_{i}$ 's, is denoted by $X_{i: n}$. A $k$-out-of- $n$ system of $n$ components functions if at least $k$ of $n$ components function. The time of a $k$-out-of- $n$ system of $n$ components with life times $X_{1}, \ldots, X_{n}$ corresponds to the $(n-k+1)$ th order statistic. Thus, the study of lifetimes of $k$-out-of- $n$ systems is equivalent to the study of the stochastic properties of order statistics. Spacings, the differences between successive order statistics, and their functions are also important in statistics, in general, and in particular in the context of life testing and reliability models. Lot of work has been done in the literature on different aspects of order statistics and spacings. For a glimpse of this, see the books by David (1981), and Arnold, Balakrishnan and Nagaraja (1992); and two volumes of papers on this topic by Balakrishnan and Rao (1998 a and b). But most of this work has been confined to the case when the observations are i.i.d. In many practical situations, like in reliability theory, the observations are not necessarily i.i.d. Because of the complicated nature of the problem, not much work has been done for the non i.i.d. case. Some references for this case are Sen (1970), David (1981, p.22), Shaked and Tong (1984), Bapat and Beg (1989), Boland et al. (1996), Kochar (1996), and Nappo and Spizzichino (1998), among others.

Some interesting partial ordering results on order statistics and spacings from independent but non-identically random variables have been obtained by Pledger and Proschan (1971), Proschan and Sethuraman (1976), Bapat and Kochar (1994), Boland, El-Neweihi, and Proschan (1994 ), Kochar and Kirmani (1995), Kochar and Korwar (1996), Kochar and Rojo (1996), Dykstra, Kochar, and Rojo (1997), Kochar and Ma (1999), Bon and Paltanea (1999), Kochar (1999), Khaledi and Kochar (1999), Khaledi and Kochar (2000 a,b,c), and Khaledi and Kochar (2001).

In this chapter, we discuss some newly obtained results on stochastic comparisons of order statistics and spacings. Kochar (1998) and Boland, Shaked and Shanthikumar (1998) have given comprehensive reviews on this topic upto 1998. In Section 2, we introduce the required notation and definitions. Section 3 and 4 are devoted to stochastic comparisons of order statistics in onesample and two-sample problems, respectively. In Sections 5, we discuss the stochastic ordering among spacings in one-sample problem and two sample problem. Section 6 is devoted to stochastic properties of sample range Throughout this chapter increasing means nondecreasing and decreasing means nonincreasing; and we shall be assuming that all distributions under 
study are absolutely continuous.

\section{Definitions}

Let $X$ and $Y$ be univariate random variables with distribution functions $F$ and $G$, survival functions $\bar{F}$ and $\bar{G}$, density functions $f$ and $g$; and hazard rates $r_{F}(=f / \bar{F})$ and $r_{G}(=g / \bar{G})$, respectively. Let $l_{X}\left(l_{Y}\right)$ and $u_{X}\left(u_{Y}\right)$ be the left and the right endpoints of the support of $X(Y)$.

\section{$\underline{\text { Stochastic orderings }}$}

Definition $2.1 X$ is said to be stochastically smaller than $Y$ (denoted by $X \leq_{\text {st }} Y$ ) if $\bar{F}(x) \leq$ $\bar{G}(x)$ for all $x$.

This is equivalent to saying that $E g(X) \leq E g(Y)$ for any increasing function $g$ for which expectations exist.

Definition $2.2 X$ is said to be smaller than $Y$ in hazard rate ordering (denoted by $X \leq_{h r} Y$ ) if $\bar{G}(x) / \bar{F}(x)$ is increasing in $x \in\left(-\infty, \max \left(u_{X}, u_{Y}\right)\right)$.

It is worth noting that $X \leq_{h r} Y$ is equivalent to the inequalities

$$
P[X-t>x \mid X>t] \leq P[Y-t>x \mid Y>t], \quad \text { for all } x \geq 0 \text { and } t .
$$

In other words, the conditional distributions, given that the random variables are at least of a certain size, are all stochastically ordered (in the standard sense) in the same direction. Thus, if $X$ and $Y$ represent the survival times of different models of an appliance that satisfy this ordering, one model is better (in the sense of stochastic ordering) when the appliances are new, the same appliance is better when both are one month old, and in fact is better no matter how much time has elapsed. It is clearly useful to know when this strong type of stochastic ordering holds since quantities judgements are then easy to make. In case the hazard rates exist, it is easy to see that $X \leq_{h r} Y$, if and only if, $r_{G}(x) \leq r_{F}(x)$ for every $x$. The hazard rate ordering is also known as uniform stochastic ordering in the literature.

Definition 2.3 $X$ is said to be smaller than $Y$ in likelihood ratio ordering (denoted by $X \leq_{l r}$ $Y)$ if $g(x) / f(x)$ is increasing in $x \in\left(l_{X}, u_{X}\right) \cup\left(l_{Y}, u_{Y}\right)$.

When the supports of $X$ and $Y$ have a common left end-point, we have the following chain of implications among the above stochastic orders :

$$
X \leq_{l r} Y \Rightarrow X \leq_{h r} Y \Rightarrow X \leq_{s t} Y .
$$

Definition 2.4 The random vector $\mathbf{X}=\left(X_{1}, \ldots, X_{n}\right)$ is smaller than the random vector $\mathbf{Y}=$ $\left(Y_{1}, \ldots, Y_{n}\right)$ in the multivariate stochastic order (denoted by $\mathbf{X} \stackrel{s t}{\preceq} \mathbf{Y}$ ) if $h(\mathbf{X}) \leq_{s t} h(\mathbf{Y})$ for all increasing functions $h$. 
It is easy to see that multivariate stochastic ordering implies component-wise usual stochastic ordering. For more details on stochastic orderings, see Chapters 1 and 4 of Shaked and Shanthikumar (1994).

One of the basic criteria for comparing variability in probability distributions is that of dispersive ordering. Let $F^{-1}$ and $G^{-1}$ be the right continuous inverses (quantile functions) of $F$ and $G$, respectively. We say that $X$ is less dispersed than $Y$ (denoted by $X \leq_{\text {disp }} Y$ ) if $F^{-1}(\beta)-F^{-1}(\alpha) \leq G^{-1}(\beta)-G^{-1}(\alpha)$, for all $0 \leq \alpha \leq \beta \leq 1$. From this one can easily obtain that

$$
X \leq_{\text {disp }} Y \Longleftrightarrow g(x) \leq f\left(F^{-1} G(x)\right) \quad \forall x,
$$

when the random variables $X$ and $Y$ admit densities. A consequence of $X \leq_{\text {disp }} Y$ is that $\left|X_{1}-X_{2}\right| \leq_{s t}\left|Y_{1}-Y_{2}\right|$ and which in turn implies $\operatorname{var}(X) \leq \operatorname{var}(Y)$ as well as $E\left[\left|X_{1}-X_{2}\right|\right] \leq$ $E\left[\left|Y_{1}-Y_{2}\right|\right]$, where $X_{1}, X_{2}\left(Y_{1}, Y_{2}\right)$ are two independent copies of $X(Y)$. For details, see Saunders and Moran (1978), Lewis and Thompson (1981), Deshpande and Kochar (1983), Bagai and Kochar (1986), Bartoszewicz (1986, 1987); and Section 2.B of Shaked and Shanthikumar (1994).

\section{Notions of Majorization and related orderings}

One of the basic tools in establishing various inequalities in statistics and probability is the notion of majorization.

Let $\left\{x_{(1)} \leq x_{(2)} \leq \ldots \leq x_{(n)}\right\}$ denote the increasing arrangement of the components of the vector $\mathbf{x}=\left(x_{1}, x_{2}, \ldots, x_{n}\right)$.

Definition 2.5 The vector $\mathbf{x}$ is said to majorize the vector $\mathbf{y}$ (written $\mathbf{x} \stackrel{m}{\succeq} \mathbf{y}$ ) if $\sum_{i=1}^{j} x_{(i)} \leq$ $\sum_{i=1}^{j} y_{(i)}$ for $j=1, \ldots, n-1$ and $\sum_{i=1}^{n} x_{(i)}=\sum_{i=1}^{n} y_{(i)}$.

Functions that preserve the majorization ordering are called Schur-convex functions. The vector $\mathbf{x}$ is said to majorize the vector $\mathbf{y}$ weakly (written $\mathbf{x} \succeq \mathbf{y}$ ) if $\sum_{i=1}^{j} x_{(i)} \leq \sum_{i=1}^{j} y_{(i)}$ for $j=1, \ldots, n$. Marshall and Olkin (1979) provides extensive and comprehensive details on the theory of majorization and its applications in statistics.

Recently Bon and Paltanea (1999) have considered a pre-order on $\mathbb{R}^{+n}$, which they call as a $p$-larger order.

Definition $2.6 A$ vector $\mathbf{x}$ in $\mathbb{R}^{+n}$ is said to be p-larger than another vector $\mathbf{y}$ also in $\mathbb{R}^{+n}$ (written $\mathbf{x} \succeq \mathbf{p}$ ) if $\prod_{i=1}^{j} x_{(i)} \leq \prod_{i=1}^{j} y_{(i)}, j=1, \ldots, n$.

Let $\log (\mathbf{x})$ denote the vector of logarithms of the coordinates of $\mathbf{x}$. It is easy to verify that

$$
\mathbf{x} \succeq \mathbf{p} \mathbf{y} \Leftrightarrow \log (\mathbf{x}) \succeq \operatorname{w} \log (\mathbf{y}) .
$$

It is known that $\mathbf{x} \succeq \stackrel{m}{\succeq} \mathbf{y} \Longrightarrow\left(g\left(x_{1}\right), \ldots, g\left(x_{n}\right)\right) \stackrel{w}{\succeq}\left(g\left(y_{1}\right), \ldots, g\left(y_{n}\right)\right)$ for all concave functions $g$ (cf. Marshal and Olkin, 1979, p. 115). From this and (2.3), it follows that when $\mathbf{x}, \mathbf{y} \in \mathbb{R}^{+^{n}}$

$$
\mathbf{x} \stackrel{m}{\succeq} \mathbf{y} \Longrightarrow \mathbf{x} \stackrel{p}{\succeq} \mathbf{y}
$$


The converse is, however, not true. For example, the vectors $(0.2,1,5) \stackrel{p}{\succeq}(1,2,3)$ but majorization does not hold between these two vectors.

\section{Notions of Aging}

Let $X$ be a random variable with distribution function $F$ and let $X_{t}$ denote a random variable with the same distribution as that of $X-t \mid X>t$. We will use the following notions of aging in this article.

(a) $X$ is said to have an increasing failure rate (denoted by $I F R$ ) distribution if $X_{t} \leq_{s t} X_{t^{\prime}}$, for $t>t^{\prime}$. This is equivalent to saying that $\bar{F}(x+t) / \bar{F}(t)$ decreasing in $t$ for $x>0$. It is easy to see that in case the random variable $X$ admits density, $F$ is $I F R$ if and only if, the hazard rate $r_{F}(t)=f(t) / \bar{F}(t)$ is increasing in $t$.

(b) $X$ is said to have a decreasing failure rate (denoted by $D F R$ ) distribution if $X_{t} \geq_{s t} X_{t^{\prime}}$, for $t>t^{\prime}$. This is equivalent to $\bar{F}(x+t) / \bar{F}(t)$ increasing in $t$ for $x>0$.

Next theorem due to Bagai and Kochar (1986) and Bartoszewicz (1987) establishes a connection between dispersive ordering and hazard rate ordering.

TheOrem 2.1 Let $X$ and $Y$ be random variables with distribution function $F$ and $G$, respectively. Then,

(a) $X \leq_{h r} Y$ and $F$ or $G$ being $D F R$ implies $X \leq_{d i s p} Y$;

(b) $X \leq_{\text {disp }} Y$ and $F$ or $G$ being IFR implies $X \leq_{h r} Y$.

\section{Stochastic Comparisons of Order Statistics in one-sample problem}

Let $X_{1}, \ldots, X_{n}$ be a set of independent random variables. It is easy to see that $X_{i: n} \leq_{s t} X_{j: n}$, for all $i<j$. Boland, El-Neweihi and Proschan (1994) extended this result from usual stochastic order to hazard rate order. Using the definition of likelihood ratio ordering, it is not hard to prove that $X_{i: n} \leq_{l r} X_{j: n}$ for $i<j$. Shaked and Shanthikumar (1994) considered the problem of comparing order statistics from samples with possibly unequal sample sizes. They showed that if random variables $X_{i}$ 's are iid, then $X_{n: n} \leq_{l r} X_{n+1: n+1}$ and $X_{1: n} \geq_{l r} X_{1: n+1}$. Raqab and Amin (1996) strengthened this result and proved that $X_{i: n} \leq_{l r} X_{j: m}$, whenever $i \leq j$ and $n-i \geq m-j$. Using implications (2.1), we get, for $i \leq j$ and $n-i \geq m-j, X_{i: n} \leq_{h r} X_{j: m}$ which in turn implies that $X_{i: n} \leq_{s t} X_{j: m}$. Removing the identically distributed assumption, it is interesting to investigate the above stochastic inequalities among order statistics. Boland, El-Neweihi and Proschan (1994) showed that if random variables are independent and $X_{k} \leq_{h r}$ $X_{n+1}, k=1, \ldots, n$, then $X_{i-1: n} \leq_{h r} X_{i: n+1}, i=1, \ldots, n+1$. They also proved that if $X_{i}$ 's are independent and $X_{n+1} \leq_{h r} X_{k}, k=2, \ldots, n$, then $X_{i: n} \geq_{h r} X_{i: n+1}, i=1, \ldots, n$. The 
reader may be wondering whether likelihood ratio ordering among order statistics holds for the case when $X_{i}$ 's are independent but not necessarily identically distributed. Assuming $X_{1} \leq_{l r} X_{2} \leq_{l r} \ldots \leq_{l r} X_{n}$, Bapat an Kochar (1994) proved that $X_{i: n} \leq_{l r} X_{j: n}, i<j$.

We end this section by discussing some results on dispersive ordering of order statistics. David and Groenveld (1982) proved that if $X_{i}$ 's are iid random variables with a common $D F R$ distribution, then $\operatorname{var}\left(X_{i: n}\right) \leq \operatorname{var}\left(X_{j: n}\right)$, for $i<j$. Kochar (1996) strengthened this result to prove that under the same conditions, $X_{i: n} \leq_{\text {disp }} X_{j: n}, i<j$. In Theorem 3.2 below, due to Khaledi and Kochar (2000 a), this result has been further extended. It is proved that if $X_{i}$ 's are iid with $D F R$ distribution, then $X_{i: n} \leq_{d i s p} X_{j: m}$, whenever $i \leq j$ and $n-i \geq m-j$. We will find the following result useful in proving it.

Theorem 3.1 (Saunders (1984)). The random variable $X$ satisfies $X \leq_{d i s p} X+Y$. for any random variable $Y$ independent of $X$ if and only if $X$ has a log-concave density.

Using Theorem 3.1, first the result is proved for exponential distribution.

LEMMA 3.1 Let $X_{i: n}$ be the ith order statistic of a random sample of size $n$ from an exponential distribution. Then

$$
X_{i: n} \leq_{d i s p} X_{j: m} \quad \text { for } i \leq j \text { and } n-i \geq m-j .
$$

Proof : Suppose we have two independent random samples, $X_{1}, \ldots, X_{n}$ and $X_{1}^{\prime}, \ldots, X_{m}^{\prime}$ of sizes $n$ and $m$ from an exponential distribution with failure rate $\lambda$. The $i t h$ order statistic $X_{i: n}$ can be written as a convolutions of the sample spacings as

$$
\begin{aligned}
X_{i: n} & =\left(X_{i: n}-X_{i-1: n}\right)+\cdots+\left(X_{2: n}-X_{1: n}\right)+X_{1: n} \\
& \stackrel{\text { dist }}{=} \sum_{k=1}^{i} E_{n-i+k}
\end{aligned}
$$

where for $k=1, \ldots, i, E_{n-i+k}$ is an exponential random variable with failure rate $(n-i+k) \lambda$. It is a well known fact that $E_{n-i+k}$ 's are independent. Similarly we can express $X_{j: m}^{\prime}$ as

$$
X_{j: m}^{\prime} \stackrel{\text { dist }}{=} \sum_{k=1}^{j} E_{m-j+k}^{\prime}
$$

where again for $k=1, \ldots, j, E_{m-j+k}^{\prime}$ is an exponential random variable with failure rate $(m-j+k) \lambda$ and $E_{m-j+k}^{\prime}$ 's are independent. It is easy to verify that $E_{n-i+1} \leq_{d i s p} E_{m-j+1}^{\prime}$ for $n-i \geq m-j$.

Since the class of distributions with log-concave densities is closed under convolutions (cf. Dharmadhiakri and Joag-dev (1988), p. 17), it follows from the repeated applications of Theorem 3.1 that

$$
\sum_{k=1}^{i} E_{n-i+k} \leq_{d i s p} \sum_{k=1}^{i} E_{m-j+k}^{\prime} .
$$


Again since $\sum_{k=i+1}^{j} E_{m-j+k}^{\prime}$, being the sum of independent exponential random variables has a $\log$-concave density and since it is independent of $\sum_{k=1}^{i} E_{n-i+k}^{\prime}$, it follows from Theorem 3.1 that the R.H.S of (3.4) is less dispersed than $\sum_{k=1}^{j} E_{m-j+k}^{\prime}$ for $i \leq j$. That is,

$$
X_{i: n} \stackrel{\text { dist }}{=} \sum_{k=1}^{i} E_{n-i+k} \leq_{d i s p} \sum_{k=1}^{j} E_{m-j+k}^{\prime} \stackrel{\text { dist }}{=} X_{j: m}^{\prime}
$$

Since $X_{j: m}$ and $X_{j: m}^{\prime}$ are stochastically equivalent, (3.1) follows from this.

The proof of the next lemma can be found in Bartoszewicz (1987).

LEMMA 3.2 Let $\phi: R_{+} \rightarrow R_{+}$be a function such that $\phi(0)=0$ and $\phi(x)-x$ is increasing. Then for every convex and strictly increasing function $\psi: R_{+} \rightarrow R_{+}$the function $\psi \phi \psi^{-1}(x)-x$ is increasing.

In the next theorem we extend Lemma 3.1 to the case when $\mathrm{F}$ is a DFR distribution.

THEOREM 3.2 Let $X_{i: n}$ be the ith order statistic of a random sample of size $n$ from a DFR distribution $F$. Then

$$
X_{i: n} \leq_{\text {disp }} X_{j: m} \quad \text { for } i \leq j \text { and } n-i \geq m-j .
$$

Proof : The distribution function of $X_{j: m}$ is $F_{j: m}(x)=B_{j: m} F(x)$, where $B_{j: m}$ is the distribution function of the beta distribution with parameters $(j, m-j+1)$.

Let $G$ denote the distribution function of a unit mean exponential random variable. Then $H_{j: m}(x)=B_{j: m} G(x)$ is the distribution function of the $j t h$ order statistic in a random sample of size $m$ from a unit mean exponential distribution. We can express $F_{j: m}$ as

$$
\begin{aligned}
F_{j: m}(x) & =B_{j: m} G G^{-1} F(x) \\
& =H_{j: m} G^{-1} F(x) .
\end{aligned}
$$

To prove the required result, we have to show that for $i \leq j$ and $n-i \geq m-j$,

$$
\begin{aligned}
& F_{j: m}^{-1} F_{i: n}(x)-x \quad \text { is increasing in } x \\
& \quad \Leftrightarrow \quad F^{-1} G H_{j: m}^{-1} H_{i: n} G^{-1} F(x)-x \quad \text { is increasing in } x .
\end{aligned}
$$

By Lemma 3.1, $H_{j: m}^{-1} H_{i: n}(x)-x$ is increasing in $x$ for $i \leq j$ and $n-i \geq m-j$. Also the function $\psi(x)=F^{-1} G(x)$ is strictly increasing and it is convex if $F$ is DFR. The required result now follows from Lemma 3.2.

REMARK: A consequence of Theorem 3.2 is that if we have random samples from a DFR distribution, then

$$
X_{i: n+1} \leq_{d i s p} X_{i: n} \leq_{d i s p} X_{i+1: n+1}, \quad \text { for } i=1, \ldots, n .
$$




\section{Stochastic Comparisons of Order Statistics in two-sample problem}

Let $X_{1}, \ldots, X_{n}$ be a set of independent random variables and $Y_{1}, \ldots, Y_{n}$ be another set of independent random variables. Ross (1983) proved that if $X_{i} \leq_{s t} Y_{i}, i=1, \ldots, n$, then $\left(X_{1}, \ldots, X_{n}\right) \leq_{s t}\left(Y_{1}, \ldots, Y_{n}\right)$. A consequence of this result is that $X_{i: n} \leq_{s t} Y_{i: n}$ for $i=$ $1, \ldots, n$. Lynch, Mimmack and Proschan (1987) generalized this result from stochastic ordering to hazard rate ordering. They showed that if $X_{i} \leq_{h r} Y_{j}, i, j \in\{1, \ldots, n\}$, then $X_{i: n} \leq_{h r} Y_{i: n}$, $i=1, \ldots, n$. A similar result for likelihood ratio ordering has been proved by Chan, Proschan and Sethuraman (1991). They proved that if $X_{i} \leq_{l r} Y_{j}, i, j \in\{1, \ldots, n\}$, then $X_{i: n} \leq_{l r} Y_{i: n}$, $i=1, \ldots, n$. Lillo, Nanda and Shaked (2000) strengthened this result to the case when the number of $X_{i}$ 's and $Y_{i}$ 's are possibly different.

Theorem 4.1 Let $X_{1}, \ldots, X_{n}$ be independent random variables and $Y_{1}, \ldots, Y_{m}$ be another set of independent random variables, all having absolutely continuous distributions. Then $X_{i} \leq_{l r} Y_{j}$ for all $i, j$ implies $X_{i: n} \leq_{l r} Y_{j: m}$ whenever $i \leq j$ and $n-i \geq m-j$.

In the next theorem we establish dispersive ordering between order statistics when the random samples are drawn from different distributions.

THEOREM 4.2 Let $X_{1}, \ldots, X_{n}$ be a random sample of size $n$ from a continuous distribution $F$ and let $Y_{1} \ldots, Y_{m}$ be a random sample of size $m$ from another continuous distribution $G$. If either $F$ or $G$ is DFR, then

$$
X \leq_{d i s p} Y \Rightarrow X_{i: n} \leq_{d i s p} Y_{j: m} \quad \text { for } i \leq j \text { and } n-i \geq m-j .
$$

Proof: Let $F$ be a $D F R$ distribution. The proof for the case when $G$ is $D F R$ is similar. By Theorem 3.2, $X_{i: n} \leq_{d i s p} X_{j: m}$ for $i \leq j$ and $n-i \geq m-j$. Bartoszewicz (1986) proved that if $X \leq_{d i s p} Y$ then $X_{j: m} \leq_{d i s p} Y_{j: m}$. Combining these we get the required result.

Since the property $X \leq_{h r} Y$ together with the condition that either $F$ or $G$ is DFR implies that $X \leq_{\text {disp }} Y$ (Theorem 2.1), we get the following result from the above theorem.

Corollary 4.1 Let $X_{1}, \ldots, X_{n}$ be a random sample of size $n$ from a continuous distribution $F$ and $Y_{1} \ldots, Y_{m}$ be a random sample of size $m$ from another continuous distribution $G$. If either $F$ or $G$ is $D F R$, then

$$
X \leq_{h r} Y \Rightarrow X_{i: n} \leq_{d i s p} Y_{j: m}
$$

\section{$\underline{\text { Stochastic comparisons of order statistics from heterogeneous populations }}$}

An assumption often made in reliability models is that the $\mathrm{n}$ components have lifetimes with proportional hazards. Let $X_{i}$ denote the lifetime of the $i t h$ component of a reliability system with survival function $\bar{F}_{i}(t), \quad i=1, \ldots, n$. Then they have proportional hazard rates (PHR) if there exist constants $\lambda_{1}, \ldots, \lambda_{n}$ and a (cumulative hazard) function $R(t) \geq 0$ such that 
$\bar{F}_{i}(t)=e^{-\lambda_{i} R(t)}$ for $i=1, \ldots, n$. Clearly then the hazard rate of $X_{i}$ is $r_{i}(t)=\lambda_{i} R^{\prime}(t)$ (assuming it exists). An example of such a situation is when the components have independent exponential lifetimes with respective hazard rates $\lambda_{1}, \ldots, \lambda_{n}$. Many researchers have investigated the effect on the survival function, the hazard rate function and other characteristics of the time to failure of this system when we switch the vector $\left(\lambda_{1}, \ldots, \lambda_{n}\right)$ to another vector say $\left(\lambda_{1}^{*}, \ldots, \lambda_{n}^{*}\right)$. Pledger and Proschan (1971), for the first time, studied this problem and proved the following interesting result among many other results.

Theorem 4.3 Let $\left(X_{1}, \ldots, X_{n}\right)$ and $\left(X_{1}^{*}, \ldots, X_{n}^{*}\right)$ be two random vectors of independent lifetimes with proportional hazards with $\lambda_{1}, \ldots, \lambda_{n}$ and $\lambda_{1}^{*}, \ldots \lambda_{n}^{*}$ as the constants of proportionality. Suppose that

$$
\lambda \stackrel{m}{\succeq} \lambda^{*}
$$

Then

$$
X_{i: n} \geq_{s t} X_{i: n}^{*}, \quad i=1, \ldots, n .
$$

Proschan and Sethuraman (1976) generalized this result from component wise stochastic ordering to multivariate stochastic ordering. That is, under the same assumptions of Theorem 4.3 , they showed that

$$
\left(X_{1: n}, \ldots, X_{n: n}\right) \geq_{s t}\left(X_{1: n}^{*}, \ldots, X_{n: n}^{*}\right) .
$$

Boland, El-Neweihi and Proschan (1994) proved that for $n=2$ the above result can be extended from stochastic ordering to hazard rate ordering. They also showed with the help of a counterexample that for $n>2,(4.2)$ cannot be strengthened from stochastic ordering to hazard rate ordering.

Dykstra, Kochar and Rojo (1997) studied the problem of stochastically comparing the largest order statistic of a set of $n$ independent and non-identically distributed exponential random variables with that corresponding to a set of $n$ independent and identically distributed exponential random variables. Let $X_{1}, \ldots, X_{n}$ be independent exponential random variables with $X_{i}$ having hazard rate $\lambda_{i}$, for $i=1, \ldots, n$. Let $Y_{1}, \ldots, Y_{n}$ be a random sample of size $n$ from an exponential distribution with common hazard rate $\bar{\lambda}=\sum_{i=1}^{n} \lambda_{i} / n$, the arithmetic mean of the $\lambda_{i}$ 's. They proved that $X_{n: n}$ is greater than $Y_{n: n}$ according to dispersive as well as hazard rate orderings. In Theorem 4.4 below we prove that similar results hold if instead, we assume that for $i=1, \ldots, n$, the random variable $Y_{i}$ has exponential distribution with hazard rate $\tilde{\lambda}=\left(\prod_{i=1}^{n} \lambda_{i}\right)^{1 / n}$, the geometric mean of the $\lambda_{i}$ 's. To prove dispersive ordering between $X_{n: n}$ and $Y_{n: n}$ in Theorem 4.4 we shall need the following lemma.

LEMMA 4.1 For $z>0$, the functions $g(z)=\left(1-e^{-z}\right) / z$ and $h(z)=\left(z^{2} e^{-z}\right) /\left(1-e^{-z}\right)^{2}$ are both decreasing.

Proof : The numerator of the derivative of $g(z)$ is $k(z)=(1+z) e^{-z}-1$, which is a decreasing function of $z$. This implies that $k(z)<0$ for $z>0$, since $k(0)=0$. 
It is easy to see after some simplifications that

$$
\frac{d}{d z}(\log (h(z)))=\frac{2-2 e^{-z}-z-z e^{-z}}{z\left(1-e^{-z}\right)} .
$$

Using the fact that $k(z)$ is negative, one can verify that the numerator of (4.3) is decreasing, from which the required result follows.

Theorem 4.4 Let $X_{1}, \ldots, X_{n}$ be independent exponential random variables with $X_{i}$ having hazard rate $\lambda_{i}, i=1, \ldots, n$. Let $Y_{1}, \ldots, Y_{n}$ be a random sample of size $n$ from an exponential distribution with common hazard rate $\tilde{\lambda}=\left(\prod_{i=1}^{n} \lambda_{i}\right)^{1 / n}$. Then

(a) $X_{n: n} \geq_{d i s p} Y_{n: n}$;

(b) $X_{n: n} \geq_{h r} Y_{n: n}$.

Proof : (a) The distribution function of $X_{n: n}$ is

$$
F_{X_{n: n}}(x)=\prod_{i=1}^{n}\left(1-e^{-\lambda_{i} x}\right)
$$

with density function as

$$
f_{X_{n: n}}(x)=\sum_{i=1}^{n} \frac{\lambda_{i} e^{-\lambda_{i} x}}{1-e^{-\lambda_{i} x}} \prod_{i=1}^{n}\left(1-e^{-\lambda_{i} x}\right) .
$$

Replacing $\lambda_{i}$ with $\tilde{\lambda}$ in (4.4), we see that the distribution function and the density function of $Y_{n: n}$ are

$$
F_{Y_{n: n}}(x)=\left(1-e^{-\tilde{\lambda} x}\right)^{n} \quad \text { and } \quad f_{Y_{n: n}}(x)=n \tilde{\lambda} e^{-\tilde{\lambda} x}\left(1-e^{-\tilde{\lambda} x}\right)^{n-1},
$$

respectively. It is easy to verify that $F_{Y_{n: n}}^{-1}(x)=-\frac{1}{\tilde{\lambda}} \log \left(1-x^{1 / n}\right)$. Using these observations, it follows that

$$
f_{Y_{n: n}}\left(F_{Y_{n: n}}^{-1} F_{X_{n: n}}(x)\right)=n \tilde{\lambda}\left(1-\prod_{i=1}^{n}\left(1-e^{-\lambda_{i} x}\right)^{1 / n}\right)\left(\prod_{i=1}^{n}\left(1-e^{-\lambda_{i} x}\right)^{1 / n}\right)^{n-1} .
$$

To prove that $X_{n: n} \geq_{\text {disp }} Y_{n: n}$, it follows from relation (2.2) that it is sufficient to show that

$$
f_{X_{n: n}}(x) \leq f_{Y_{n: n}}\left(F_{Y_{n: n}}^{-1} F_{X_{n: n}}(x)\right) \quad \forall x>0 .
$$

Using expressions (4.4) and (4.5) in (4.6), one can see after some simplifications that (4.6) is equivalent to

$$
\sum_{i=1}^{n} \frac{\lambda_{i}}{1-e^{-\lambda_{i} x}}-n \prod_{i=1}^{n}\left(\frac{\lambda_{i}}{1-e^{-\lambda_{i} x}}\right)^{1 / n} \leq \sum_{i=1}^{n} \lambda_{i}-n \prod_{i=1}^{n}\left(\lambda_{i}\right)^{1 / n} .
$$

To prove that (4.7) holds for all $\lambda_{i}>0, i=1, \ldots, n$, it is sufficient to show that the L.H.S. of (4.7) (denoted by $h(x)$ ) is increasing in $x$ since for $x>0$,

$$
h(x) \leq \lim _{x \rightarrow+\infty} h(x)=\sum_{i=1}^{n} \lambda_{i}-n \prod_{i=1}^{n}\left(\lambda_{i}\right)^{1 / n},
$$


the right hand side of (4.7).

The derivative of $h(x)$ is

$$
\begin{aligned}
h^{\prime}(x) & =\left(\sum_{i=1}^{n} \frac{\lambda_{i} e^{-\lambda_{i} x}}{1-e^{-\lambda_{i} x}}\right)\left(\prod_{i=1}^{n} \frac{\lambda_{i}}{1-e^{-\lambda_{i} x}}\right)^{1 / n}-\sum_{i=1}^{n} \frac{\lambda_{i}^{2} e^{-\lambda_{i} x}}{\left(1-e^{-\lambda_{i} x}\right)^{2}} \\
& \geq\left(\sum_{i=1}^{n} \frac{\lambda_{i} e^{-\lambda_{i} x}}{1-e^{-\lambda_{i} x}}\right)\left(\frac{n}{\sum_{i=1}^{n} \frac{1-e^{-\lambda_{i} x}}{\lambda_{i}}}\right)-\sum_{i=1}^{n} \frac{\lambda_{i}^{2} e^{-\lambda_{i} x}}{\left(1-e^{-\lambda_{i} x}\right)^{2}},
\end{aligned}
$$

since the geometric mean of a set of numbers is always greater than or equal to its harmonic mean. Now $h^{\prime}(x) \geq 0$ if and only if,

$$
n \sum_{i=1}^{n} \frac{\lambda_{i} e^{-\lambda_{i} x}}{1-e^{-\lambda_{i} x}} \geq\left(\sum_{i=1}^{n} \frac{\lambda_{i}^{2} e^{-\lambda_{i} x}}{\left(1-e^{-\lambda_{i} x}\right)^{2}}\right)\left(\sum_{i=1}^{n} \frac{1-e^{-\lambda_{i} x}}{\lambda_{i}}\right) .
$$

Multiplying both sides of (4.8) by $x(>0)$ and replacing the $\lambda_{i} x$ with $z_{i}$ for $i=1, \ldots, n$, it is enough to prove that

$$
n \sum_{i=1}^{n} \frac{z_{i} e^{-z_{i}}}{1-e^{-z_{i}}} \geq\left(\sum_{i=1}^{n} \frac{z_{i}^{2} e^{-z_{i}}}{\left(1-e^{-z_{i}}\right)^{2}}\right)\left(\sum_{i=1}^{n} \frac{1-e^{-z_{i}}}{z_{i}}\right) .
$$

The inequality in (4.9) follows immediately from Čebyšev's inequality (Theorem 1, p. 36 of Mitrinović, 1970), Lemma 4.1 and by writing

$$
\frac{z_{i} e^{-z_{i}}}{1-e^{-z_{i}}}=\left(\frac{z_{i}^{2} e^{-z_{i}}}{\left(1-e^{-z_{i}}\right)^{2}}\right)\left(\frac{1-e^{-z_{i}}}{z_{i}}\right) \text {. }
$$

This proves that $h(x)$ is increasing in $x$ and hence the result.

(b) It follows from Theorem 5.8 of Barlow and Proschan (1981) that $Y_{n: n}$ is $I F R$. Using this and part (a), the required result follows from Theorem 2.1.

From the above results, we get the following convenient bounds on the hazard rate and the variance of $X_{n: n}$.

COROLlary 4.2 Under the conditions of Theorem 4.4,

(a) the hazard rate $r_{X_{n: n}}$ of $X_{n: n}$ satisfies

$$
r_{X_{n: n}}(x ; \boldsymbol{\lambda}) \leq \frac{n \tilde{\lambda}(1-\exp (-\tilde{\lambda} x))^{n-1} \exp (-\tilde{\lambda} x)}{1-(1-\exp (-\tilde{\lambda} x))^{n}},
$$

(b)

$$
\operatorname{var}\left(X_{n: n} ; \boldsymbol{\lambda}\right) \geq \frac{1}{\tilde{\lambda}^{2}} \sum_{i=1}^{n} \frac{1}{(n-i+1)^{2}}
$$


Dykstra, Kochar and Rojo (1997) proved a result similar to Theorem 4.4 by assuming that the random variables $Y_{i}^{\prime}$ 's are exponential with common hazard rate $\bar{\lambda}=\sum_{i=1}^{n} \lambda_{i} / n$ and obtained bounds on the hazard rate and the variance of $X_{n: n}$ in terms of $\bar{\lambda}$. The new bounds given in Corollary 4.2 are better because $r_{Y_{n: n}}$ and $\operatorname{var}\left(Y_{n: n}\right)$ are increasing and decreasing function of $\tilde{\lambda}$, respectively, and the fact that the geometric mean of $\lambda_{i}$ 's is smaller than their arithmetic mean.

Figure 4.1. Graphs of hazard rates of $X_{3: 3}$

Figure 4.2. Graphs of hazard rates of $X_{3: 3}$

In Figures 4.1. and 4.2. above, we plot the hazard rates of parallel systems of three exponential components along with the upper bounds as given by Dykstra, Kochar and Rojo (1997) and the one's given by Corollary 4.2 (a). The vector of parameters in Figure 4.1 is $\boldsymbol{\lambda}_{\mathbf{1}}=(1,2,3)$ and that in Figure 4.2 is $\boldsymbol{\lambda}_{\mathbf{2}}=(0.2,2,3.8)$. Note that $\boldsymbol{\lambda}_{\mathbf{2}} \stackrel{m}{\succeq} \boldsymbol{\lambda}_{\mathbf{1}}$. It appears from these figures that the improvements in the bounds are relatively more if $\lambda_{i}$ 's are more dispersed in the sense of majorization. This is true because the geometric mean is Schur-concave and the 
hazard rate of a parallel system of i.i.d. exponential components with a common parameter $\tilde{\lambda}$ is increasing in $\tilde{\lambda}$.

Let $\bar{F}$ denote the survival function of a nonnegative random variable $X$ with hazard rate $h$. According to the PHR model, the random variables $X_{1}, \ldots, X_{n}$ are independent with $X_{i}$ having survival function $\bar{F}^{\lambda_{i}}($.$) , so that its hazard rate is \lambda_{i} h(),. \quad i=1, \ldots, n$.

Next, we extend Theorem 4.4 from exponential to PHR models. To prove this we need the following theorem due to Rojo and He (1991).

Theorem 4.5 Let $X$ and $Y$ be two random variables such that $X \leq_{s t} Y$. Then $X \leq_{\text {disp }} Y$ implies that $\gamma(X) \leq_{\text {disp }} \gamma(Y)$ where $\gamma$ is a nondecreasing convex function.

TheOREM 4.6 Let $X_{1}, \ldots, X_{n}$ be independent random variables with $X_{i}$ having survival function $\bar{F}^{\lambda_{i}}(x), \quad i=1, \ldots, n$. Let $Y_{1}, \ldots, Y_{n}$ be a random sample of size $n$ from a distribution with survival function $\bar{F}^{\tilde{\lambda}}(x)$, where $\tilde{\lambda}=\left(\prod_{i=1}^{n} \lambda_{i}\right)^{1 / n}$. Then

(a) $X_{n: n} \geq_{h r} Y_{n: n}$; and

(b) if $F$ is DFR, then $X_{n: n} \geq_{\text {disp }} Y_{n: n}$.

Proof : (a)

Let $H(x)=-\log \bar{F}(x)$ denote the cumulative hazard of $F$. Let $Z_{i}=H\left(X_{i}\right), i=1, \ldots, n$ and $W_{i}=H\left(Y_{i}\right), i=1, \ldots, n$. Since $X_{i}$ 's follow the PHR model, then it is easy to show that $Z_{i}$ is exponential with hazard rate $\lambda_{i}, i=1, \ldots, n$. Similarly, $W_{i}$ is exponential with hazard rate $\tilde{\lambda}, i=1, \ldots, n$. Theorem 4.4 (b) implies that $Z_{n: n} \geq_{h r} W_{n: n}$. Using this fact, (since $H^{-1}$, the right inverse of $H$, is nondecreasing) it is easy to show that $H^{-1}\left(Z_{n: n}\right) \geq_{h r} H^{-1}\left(W_{n: n}\right)$ from which the part (a) follows.

(b) Theorem 4.4 (a) and (b), respectively, imply that $Z_{n: n} \geq_{\text {disp }} W_{n: n}$ and $Z_{n: n} \geq_{s t} W_{n: n}$. The function $H^{-1}(x)$ is convex, since $F$ is $D F R$, and is nondecreasing. Using these observations, it follows from Theorem 4.5 that $H^{-1}\left(Z_{n: n}\right) \geq_{\text {disp }} H^{-1}\left(W_{n: n}\right)$ which is equivalent to $X_{n: n} \geq_{\text {disp }} Y_{n: n}$.

In Theorem 4.9 below we prove that for the largest order statistic, the conclusion of Theorem 4.3 holds under the weaker $p$-larger ordering. The proof of this theorem hinges on the following results.

Theorem 4.7 (Marshall and Olkin, 1979, p. 57) Let $I \subset \mathbb{R}$ be an open interval and let $\phi: I^{n} \rightarrow \mathbb{R}$ be continuously differentiable. Necessary and sufficient conditions for $\phi$ to be Schur-convex on $I^{n}$ are $\phi$ is symmetric on $I^{n}$ and for all $i \neq j$,

$$
\left(z_{i}-z_{j}\right)\left[\phi_{(i)}\left(z_{i}\right)-\phi_{(j)}\left(z_{j}\right)\right] \geq 0 \quad \text { for all } z \in I^{n},
$$

where $\phi_{(i)}(z)$ denotes the partial derivative of $\phi$ with respect to its ith argument. 
Theorem 4.8 (Marshall and Olkin, 1979, p. 59) A real-valued function $\phi$ on the set $A \subset \mathbb{R}^{n}$ satisfies

$$
\mathbf{x} \succeq^{w} \mathbf{y} \text { on } A \Longrightarrow \phi(\mathbf{x}) \geq \phi(\mathbf{y})
$$

if and only if $\phi$ is decreasing and Schur-convex on $A$.

LEMMA 4.2 The function $\psi: \mathbb{R}^{+^{n}} \rightarrow \mathbb{R}$ satisfies

$$
\mathbf{x} \stackrel{p}{\succeq} \mathbf{y} \Longrightarrow \psi(\mathbf{x}) \geq \psi(\mathbf{y})
$$

if and only if,

(i) $\psi\left(e^{a_{1}}, \ldots, e^{a_{n}}\right)$ is Schur-convex in $\left(a_{1}, \ldots, a_{n}\right)$

(ii) $\psi\left(e^{a_{1}}, \ldots, e^{a_{n}}\right)$ is decreasing in $a_{i}$, for $i=1, \ldots, n$,

where $a_{i}=\log x_{i}$, for $i=1, \ldots, n$.

Proof : Using relation (2.3), we see that (4.10) is equivalent to

$$
\mathbf{a} \succeq \mathbf{w} \Longrightarrow \psi\left(e^{a_{1}}, \ldots, e^{a_{n}}\right) \geq \psi\left(e^{b_{1}}, \ldots, e^{b_{n}}\right),
$$

where $a_{i}=\log x_{i}$ and $b_{i}=\log y_{i}$, for $i=1, \ldots, n$.

Taking $\phi\left(a_{1}, \ldots, a_{n}\right)=\psi\left(e^{a_{1}}, \ldots, e^{a_{n}}\right)$ in Theorem 4.8 , we get the required result.

Now we are ready to prove the next theorem.

THeOREM 4.9 Let $X_{1}, \ldots, X_{n}$ be independent random variables with $X_{i}$ having survival function $\bar{F}^{\lambda_{i}}(x), \quad i=1, \ldots, n$. Let $Y_{1}, \ldots, Y_{n}$ be another set of random variables with $Y_{i}$ having survival function $\bar{F}^{\lambda_{i}^{*}}(x), i=1, \ldots, n$. Then

$$
\boldsymbol{\lambda} \stackrel{p}{\succeq} \lambda^{*} \Longrightarrow X_{n: n} \geq_{s t} Y_{n: n} .
$$

Proof : The survival function of $X_{n: n}$ can be written as

$$
\bar{F}_{X_{n: n}}(x)=1-\prod_{i=1}^{n}\left(1-e^{-e^{a_{i}} H(x)}\right),
$$

where $a_{i}=\log \lambda_{i}, i=1, \ldots, n$ and $H(x)=-\log \bar{F}(x)$.

Using Lemma 4.2, we find that it is enough to show that the function $\bar{F}_{X_{n: n}}$ given by (4.12) is Schur-convex and decreasing in $a_{i}$ 's. To prove its Schur-convexity, it follows from Theorem 4.7 that, we have to show that for $i \neq j,\left(a_{i}-a_{j}\right)\left(\frac{\partial \bar{F}_{X_{n: n}}}{\partial a_{i}}-\frac{\partial \bar{F}_{X_{n: n}}}{\partial a_{j}}\right) \geq 0$. That is,

$$
H(x)\left(a_{i}-a_{j}\right)\left(\prod_{i=1}^{n}\left(1-e^{-e^{a_{i}} H(x)}\right)\right)\left(\frac{e^{a_{j}} e^{-e^{a_{j}} H(x)}}{1-e^{-e^{a_{j}} H(x)}}-\frac{e^{a_{i}} e^{-e^{a_{i}} H(x)}}{1-e^{-e^{a_{i}} H(x)}}\right) \geq 0, \text { for } i \neq j
$$

since

$$
\frac{\partial \bar{F}_{X_{n: n}}}{\partial a_{i}}=-\prod_{i=1}^{n}\left(1-e^{-e^{a_{i}} H(x)}\right)\left(\frac{H(x) e^{a_{i}} e^{-e^{a_{i}} H(x)}}{1-e^{-e^{a_{i}} H(x)}}\right) .
$$


It is easy to see that the function $b e^{-b H(x)} /\left(1-e^{-b H(x)}\right)$ is decreasing in $b$, for each fixed $x>0$. Replacing $b$ with $e^{a_{i}}$, it follows that the function $e^{a_{i}} e^{-e^{a_{i}} H(x)} /\left(1-e^{-e^{a_{i}} H(x)}\right)$ is also decreasing in $a_{i}$ for $i=1, \ldots, n$. This proves that (4.13) holds. The partial derivative of $\bar{F}_{X_{n: n}}$ with respect to $a_{i}$ is negative and which in turn implies that the survival function of $X_{n: n}$ is decreasing in $a_{i}$ for $i=1, \ldots, n$. This completes the proof.

The following result due to Khaledi and Kochar (2000 b) is a special case of Theorem 4.9.

Corollary 4.3 Let $X_{1}, \ldots, X_{n}$ be independent exponential random variables with $X_{i}$ having hazard rate $\lambda_{i}, i=1, \ldots, n$. Let $Y_{1}, \ldots, Y_{n}$ be another set of independent exponential random variables with $Y_{i}$ having hazard rate $\lambda_{i}^{*}, i=1, \ldots, n$. Then

$$
\boldsymbol{\lambda} \stackrel{p}{\succeq} \boldsymbol{\lambda}^{*} \Longrightarrow X_{n: n} \geq_{s t} Y_{n: n}
$$

Boland, El-Neweihi and Proschan (1994 ) pointed out that for $n>2$, (4.2) cannot be strengthened from stochastic ordering to hazard rate ordering. Since majorization implies $p$ larger ordering, it follows that, in general, Theorem 4.9 cannot be strengthened to hazard rate ordering.

As shown in the next example, a result similar to Theorem 4.9 may not hold for other order statistics.

EXAMPLE 4.1: Let $X_{1}, X_{2}, X_{3}$ be independent exponential random variables with $\boldsymbol{\lambda}=$ $(0.1,1,7.9)$ and $Y_{1}, Y_{2}, Y_{3}$ be independent exponential random variables with $\boldsymbol{\lambda}^{*}=(1,2,5)$. It is easy to see that $\boldsymbol{\lambda} \succeq \boldsymbol{\lambda}^{*}$. The $X_{1: 3}$ and $Y_{1: 3}$ have exponential distributions with respective hazard rates 9 and 8 and which implies that $Y_{1: 3} \geq_{s t} X_{1: 3}$.

\section{Stochastic Comparisons of Sample Spacings}

Let $X_{1}, \ldots, X_{n}$ be $n$ random variables. The random variables $D_{i: n}=X_{i: n}-X_{i-1: n}$ and $D_{i: n}^{*}=(n-i+1) D_{i: n}, i=1, \ldots, n$, with $X_{0: n} \equiv 0$, are respectively called spacings and normalized spacings. They are of great interest in various areas of statistics, in particular, in characterizations of distributions, goodness-of-fit tests, life testing and reliability models. In the reliability context they correspond to times elapsed between successive failures of components in a system. It is well known that the normalized spacings of a random sample from an exponential distribution are i.i.d. random variables having the same exponential distribution. Such a characterization may not hold for other distributions and much of the reliability theory deals with this aspect of spacings. In this section we review stochastic properties of spacings when original random variables are i.i.d. as well as when they are independent but not identically distributed.

Many authors have studied the stochastic properties of spacings from restricted families of distributions. Barlow and Proschan (1966) proved that if $X_{1}, \ldots, X_{n}$ is a random sample from a $D F R$ distribution, then the successive normalized spacings are stochastically increasing. 
Kochar and Kirmani (1995) strengthened this result from stochastic ordering to hazard rate ordering, that is, for $i=1, \ldots, n-1$,

$$
D_{i: n}^{*} \leq_{h r} D_{i+1: n}^{*}
$$

The corresponding problem when the random variables are not identically distributed, has also been studied by many researchers, including Pledger and Proschan (1971), Shaked and Tong (1984), Kochar and Korwar (1996), Kochar and Rojo (1996), Nappo and Spizzichino (1998), among others. For a review of this topic see Kochar (1998). Here we give some new results obtained recently by the authors.

Kochar and Korwar (1996) conjectured that a result similar to (5.1) holds in the case when $X_{1}, \ldots, X_{n}$ are independent exponential random variables with $X_{i}$ having hazard rate $\lambda_{i}$, for $i=1, \ldots, n$. Khaledi and Kochar $(2001)$ proved this conjecture when random variables $X_{i}$ 's follow a single outlier model with parameters $\lambda$ and $\lambda^{*}$, that is when $\lambda_{1}=\ldots=\lambda_{n-1}=\lambda$ and $\lambda_{n}=\lambda^{*}$. To prove this we shall be using the following results.

The joint density function of the spacings when $\lambda_{i}$ 's are possibly different is given by (cf. Kochar and Korwar, 1996),

$$
f_{D_{1: n}, \ldots, D_{n: n}}\left(x_{1}, \ldots, x_{n}\right)=\sum_{(\mathbf{r})} \frac{\prod_{i=1}^{n} \lambda_{i}}{\prod_{i=1}^{n} \sum_{j=i}^{n} \lambda\left(r_{j}\right)} \prod_{i=1}^{n}\left(\sum_{j=i}^{n} \lambda\left(r_{j}\right)\right) \exp \left\{-x_{i} \sum_{j=i}^{n} \lambda\left(r_{j}\right)\right\},
$$

for $x_{i} \geq 0, i=1, \ldots, n$, where $(\mathbf{r})=\left(r_{1}, \ldots, r_{n}\right)$ is a permutation of $(1, \ldots, n)$ and $\lambda(i)=\lambda_{i}$. It is a mixture of products of exponential random variables. From (5.2) it is easy to find that the joint pdf of $\left(D_{i: n}, D_{j: n}\right)$ for $1 \leq i<j \leq n$, is

$$
\begin{aligned}
f_{D_{i: n}, D_{j: n}}(x, y) & =\sum_{(\mathbf{r})} \frac{\prod_{i=1}^{n} \lambda_{i}}{\prod_{i=1}^{n} \sum_{j=i}^{n} \lambda\left(r_{j}\right)} \\
& \times\left(\sum_{m=i}^{n} \lambda\left(r_{m}\right)\right) \exp \left\{-x \sum_{m=i}^{n} \lambda\left(r_{m}\right)\right\}\left(\sum_{m=j}^{n} \lambda\left(r_{m}\right)\right) \exp \left\{-y \sum_{m=j}^{n} \lambda\left(r_{m}\right)\right\},
\end{aligned}
$$

for $x, y \geq 0$. Now (5.2) can be written as

$$
\begin{gathered}
f_{D_{1: n}, \ldots, D_{n: n}}\left(x_{1}, \ldots, x_{n}\right)=\sum_{\theta=1}^{n} \frac{(n-1) ! \lambda^{*}(\lambda)^{n-1}}{\prod_{i=1}^{\theta}\left((n-i) \lambda+\lambda^{*}\right) \prod_{i=\theta+1}^{n}(n-i+1) \lambda} \\
\times \prod_{i=1}^{\theta}\left((n-i) \lambda+\lambda^{*}\right) e^{-\left((n-i) \lambda+\lambda^{*}\right) x_{i}} \prod_{i=\theta+1}^{n}(n-i+1) \lambda e^{-(n-i+1) \lambda x_{i}},
\end{gathered}
$$

which can be further expressed as

$$
f_{D_{1: n}, \ldots, D_{n: n}}\left(x_{1}, \ldots, x_{n}\right)=\sum_{\theta=1}^{n} h(\theta) \prod_{i=1}^{\theta} \alpha_{i}^{*} e^{-\alpha_{i}^{*} x_{i}} \prod_{i=\theta+1}^{n} \alpha_{i} e^{-\alpha_{i} x_{i}},
$$

where $\alpha_{i}=(n-i+1) \lambda, \alpha_{i}^{*}=(n-i) \lambda+\lambda^{*}, i=1, \ldots, n$ and using $\alpha_{i}$ and $\alpha_{i}^{*}$, the function $h$ is given by

$$
h(\theta)=\frac{(n-1) ! \lambda^{n-1} \lambda^{*}}{\prod_{i=1}^{\theta} \alpha_{i}^{*} \prod_{i=\theta+1}^{n} \alpha_{i}}, \quad \theta=1, \ldots, n .
$$


The marginal density function of $D_{i: n}$ can be expressed as

$$
f_{D_{i: n}}(x)=H_{i} \alpha_{i} e^{-\alpha_{i} x}+\bar{H}_{i} \alpha_{i}^{*} e^{-\alpha_{i}^{*} x},
$$

where

$$
H_{i}=\sum_{\theta=1}^{i-1} h(\theta), \quad i=2, \ldots, n \text { and } H_{1}=0 .
$$

Thus, the density function of $D_{i: n}$ is a mixture of two exponential random variables with parameters $\alpha_{i}$ and $\alpha_{i}^{*}$. Now we prove the main theorem.

Theorem 5.1 Let $X_{1}, \ldots, X_{n}$ follow the single-outlier exponential model with parameters $\lambda$ and $\lambda^{*}$. Then

$$
D_{i+1: n}^{*} \geq_{h r} D_{i: n}^{*}, \quad i=1, \ldots, n-1 .
$$

Proof : We prove the result when $\lambda^{*}>\lambda$. The proof for the case $\lambda^{*}<\lambda$ follows using the same kind of arguments. From (5.6) we find that the survival function of $D_{i: n}^{*}$ is $\bar{F}_{D_{i: n}^{*}}(x)=$ $H_{i} e^{-\lambda x}+\bar{H}_{i} e^{-\eta_{i} x}$, where $\eta_{i}=\frac{(n-i) \lambda+\lambda^{*}}{n-i+1}$. To prove the theorem we have to show that for any $i \in\{1, \ldots, n-1\}$,

$$
g(x)=\frac{\bar{F}_{D_{i+1: n}^{*}}(x)}{\bar{F}_{D_{i: n}^{*}}(x)}
$$

is increasing in $x$. The numerator of $g^{\prime}(x)$, the derivative of $g(x)$ is

$$
\begin{aligned}
A(x)= & {\left[H_{i} e^{-\lambda x}+\bar{H}_{i} e^{-\eta_{i} x}\right]\left[-\lambda H_{i+1} e^{-\lambda x}-\eta_{i+1} \bar{H}_{i+1} e^{-\eta_{i+1} x}\right] } \\
& +\left[H_{i+1} e^{-\lambda x}+\bar{H}_{i+1} e^{-\eta_{i+1} x}\right]\left[\lambda H_{i} e^{-\lambda x}+\eta_{i} \bar{H}_{i} e^{-\eta_{i} x}\right] \\
= & \left(\lambda^{*}-\lambda\right)\left\{\frac{\bar{H}_{i} H_{i+1}}{n-i+1} e^{-\left(\eta_{i}+\lambda\right) x}\right. \\
& \left.-\frac{\bar{H}_{i+1} H_{i}}{n-i} e^{-\left(\eta_{i+1}+\lambda\right) x}-\frac{\bar{H}_{i} \bar{H}_{i+1}}{(n-i+1)(n-i)} e^{\left(\eta_{i}+\eta_{i+1}\right) x}\right\} \\
\geq & \left(\lambda^{*}-\lambda\right)\left\{\left(\frac{\bar{H}_{i} H_{i+1}}{n-i+1}-\frac{\bar{H}_{i+1} H_{i}}{n-i}\right) e^{-\left(\eta_{i+1}+\lambda\right) x}\right. \\
& \left.-\frac{\bar{H}_{i} \bar{H}_{i+1}}{(n-i+1)(n-i)} e^{\left(\eta_{i}+\eta_{i+1}\right) x}\right\} \\
= & \frac{\left(\lambda^{*}-\lambda\right)}{(n-i)(n-i+1)}\left\{\left\{(n-i) \bar{H}_{i}-(n-i+1) \bar{H}_{i+1}+\bar{H}_{i} \bar{H}_{i+1}\right\}\right. \\
\times & \left.e^{-\left(\eta_{i+1}+\lambda\right) x}-\bar{H}_{i} \bar{H}_{i+1} e^{-\left(\eta_{i}+\eta_{i+1}\right) x}\right\} .
\end{aligned}
$$

The inequality in (5.8) follows, since $\lambda^{*}>\lambda$ implies $\eta_{i+1}>\eta_{i}$.

Again $\lambda^{*}>\lambda$ implies $\lambda<\eta_{i}$ and which in turn implies $e^{-\left(\eta_{i+1}+\lambda\right) x} \geq e^{-\left(\eta_{i}+\eta_{i+1}\right) x}$ for every $x \geq 0$. Also for $\lambda^{*}>\lambda$,

$$
\begin{aligned}
\left\{(n-i) \bar{H}_{i}-(n-i+1) \bar{H}_{i+1}\right\} & =(n-i) h(i)-\bar{H}_{i+1} \\
& \geq 0,
\end{aligned}
$$


since for $\lambda^{*}>\lambda, h(j)$ is a decreasing function of $j$. Using these results in (5.9) we find that $A(x)$ and hence $g^{\prime}(x)$ is nonnegative for $x \geq 0$. This proves the required result.

Let $X_{1}, \ldots, X_{n}$ be independent exponential random variables with hazard rates $\lambda_{1}, \ldots, \lambda_{n}$, respectively. Pledger and Proschan (1971) proved that for $i \in\{1, \ldots, n\}, D_{i: n}$ is stochastically larger when the hazard rates are unequal than when they are all equal. Kochar and Rojo (1996) strengthened this result to likelihood ratio ordering. The natural question is to examine whether the survival function of $D_{i: n}$ is Schur-convex in $\left(\lambda_{1}, \ldots, \lambda_{n}\right)$. Pledger and Proschan (1971) came up with a counterexample to show that this is not true in general. Kochar and Korwar (1996) proved that in the special case of second spacing, whereas the survival function of $D_{2: n}$ is Schur-convex in $\left(\lambda_{1}, \ldots, \lambda_{n}\right)$, its hazard rate is not Schur-concave. They proved, however, that the hazard rate of $D_{2: 2}$ is Schur-concave. We now examine this question when $X_{1}, \ldots, X_{n}$ follow the single-outlier exponential model with parameters $\lambda$ and $\lambda^{*}$. In the rest of this section, we assume that $\lambda^{*}<\lambda$. We will treat it as a part of the model. It is easy to see that in this case, $\left(\lambda_{1}^{*}, \lambda_{1}, \ldots, \lambda_{1}\right) \succeq\left(\lambda_{2}^{*}, \lambda_{2}, \ldots, \lambda_{2}\right)$ if and only if $\lambda_{1}^{*}<\lambda_{2}^{*}<\lambda_{2}<\lambda_{1}$ and $\lambda_{1}^{*}+(n-1) \lambda_{1}=\lambda_{2}^{*}+(n-1) \lambda_{2}$. We prove later in this section that for the single-outlier model, for $i \in\{1, \ldots, n\}$, the hazard rate of $D_{i: n}$ is Schur-concave in $\lambda$ 's. To prove it we need the following lemmas.

LEMMA 5.1 Let $X_{1}, \ldots, X_{n}$ follow the single-outlier exponential model with parameters $\lambda$ and $\lambda^{*}$. Then

$$
\lambda^{*}<\lambda \Longrightarrow H_{i} \leq \frac{i-1}{n}, \text { for } i=1, \ldots, n,
$$

where $H_{i}$ is given by (5.7). The inequality in (5.11) is reversed for $\lambda^{*}>\lambda$.

Proof : $\quad \lambda^{*}<\lambda$ implies that the function $h(j)$ in (5.5) is increasing in $j, j=1, \ldots, n$. Note that

$$
(h(1), h(2), \ldots, h(n)) \stackrel{m}{\succeq}(1 / n, \ldots, 1 / n) .
$$

The required result follows from the definition of majorization.

The proof for the case $\lambda^{*}>\lambda$ follows from the same kind of arguments.

LEMMA 5.2 Let $X_{1}, \ldots, X_{n}$ follow the single-outlier exponential model with parameters $\lambda_{1}$ and $\lambda_{1}^{*}$. Let $Y_{1}, \ldots, Y_{n}$ be another set of random variables following the single-outlier exponential model with parameters $\lambda_{2}$ and $\lambda_{2}^{*}$. If

(i) $\lambda_{1}^{*}<\lambda_{2}^{*}<\lambda_{2}<\lambda_{1}$, then $\Theta_{1} \geq_{l r} \Theta_{2}$,

(ii) $\lambda_{1}<\lambda_{2}<\lambda_{2}^{*}<\lambda_{1}^{*}$, then $\Theta_{1} \leq_{l r} \Theta_{2}$,

where $\Theta_{1}$ and $\Theta_{2}$ correspond to random variable $\Theta$ with probability mass function $h(j)$ in $(5.5)$ for $X_{i}$ 's and $Y_{i}$ 's, respectively.

Proof : (i) We prove that

$$
\frac{h_{2}(\theta+1)}{h_{1}(\theta+1)} \leq \frac{h_{2}(\theta)}{h_{1}(\theta)}
$$


where $h_{1}$ and $h_{2}$ are probability mass functions of $\Theta_{1}$ and $\Theta_{2}$, respectively. This inequality holds if and only if

$$
\frac{(n-\theta-1) \lambda_{1}+\lambda_{1}^{*}}{(n-\theta-1) \lambda_{2}+\lambda_{2}^{*}} \leq \frac{\lambda_{1}}{\lambda_{2}}
$$

Since $\lambda_{1}^{*}<\lambda_{2}^{*}$ and $\lambda_{2}<\lambda_{1}$, it is easy to see that (5.12) is true.

(ii) In this case the inequality in (5.12) is reversed which in turn implies that $\Theta_{1} \leq_{l r} \Theta_{2}$. This proves the result.

Theorem 5.2 Let $X_{1}, \ldots, X_{n}$ follow the single-outlier exponential model with parameters $\lambda_{1}$ and $\lambda_{1}^{*}$ with $\lambda_{1}^{*}<\lambda_{1}$. Then for $i \in\{1, \ldots, n\}$, the hazard rate of $D_{i: n}$ is Schur-concave in $\left\{\lambda_{1}, \ldots, \lambda_{1}, \lambda_{1}^{*}\right\}$.

Proof : Let $Y_{1}, \ldots, Y_{n}$ be another set of random variables following the single-outlier exponential model with parameters $\lambda_{2}$ and $\lambda_{2}^{*}\left(\lambda_{2}^{*}<\lambda_{2}\right)$ such that $\left(\lambda_{1}^{*}, \lambda_{1}, \ldots, \lambda_{1}\right) \stackrel{m}{\succeq}\left(\lambda_{2}^{*}, \lambda_{2}, \ldots, \lambda_{2}\right)$. As discussed above this holds if and only if $\lambda_{1}^{*}<\lambda_{2}^{*}<\lambda_{2}<\lambda_{1}$ and $\lambda_{1}^{*}+(n-1) \lambda_{1}=\lambda_{2}^{*}+(n-1) \lambda_{2}$. Without loss of generality, let us assume that $\lambda_{1}^{*}+(n-1) \lambda_{1}=1$. We have to prove that under the given conditions for $i=1, \ldots, n$,

$$
D_{i: n}^{(1)} \geq_{h r} D_{i: n}^{(2)}
$$

where $D_{i: n}^{(1)}\left(D_{i: n}^{(2)}\right)$ denotes the $i$ th spacing of $X_{i}$ 's $\left(Y_{i}\right.$ 's). From (5.6) the survival functions of $D_{i: n}^{(1)}$ and $D_{i: n}^{(2)}$ are

$$
\begin{aligned}
& \bar{F}_{D_{i: n}^{(1)}}(x)=P_{i} e^{-\alpha_{i 1} x}+\bar{P}_{i} e^{-\alpha_{i 1}^{*} x}, \\
& \bar{F}_{D_{i: n}^{(2)}}(x)=Q_{i} e^{-\alpha_{i 2} x}+\bar{Q}_{i} e^{-\alpha_{i 2}^{*} x},
\end{aligned}
$$

where $P_{i}$ and $Q_{i}$ correspond to $H_{i}$ in (5.6) for $D_{i: n}^{(1)}$ and $D_{i: n}^{(2)}$, respectively and $\alpha_{i 1}=(n-i+1) \lambda_{1}$, $\alpha_{i 1}^{*}=(n-i) \lambda_{1}+\lambda_{1}^{*}, \alpha_{i 2}=(n-i+1) \lambda_{2}$ and $\alpha_{i 2}^{*}=(n-i) \lambda_{2}+\lambda_{2}^{*}$.

We have to show that

$$
\phi(x)=\frac{\bar{F}_{D_{i: n}^{(1)}}(x)}{\bar{F}_{D_{i: n}^{(2)}}(x)}
$$

is increasing in $x$. After some simplifications we find that the numerator of $\phi^{\prime}(x)$, the derivative of $\phi(x)$ is

$$
\begin{aligned}
g(x) & =-\left(\alpha_{i 1}-\alpha_{i 2}\right) P_{i} Q_{i} e^{-\left(\alpha_{i 1}+\alpha_{i 2}\right) x}+\left(\alpha_{i 2}^{*}-\alpha_{i 1}^{*}\right) \bar{P}_{i} \bar{Q}_{i} e^{-\left(\alpha_{i 1}^{*}+\alpha_{i 2}^{*}\right) x} \\
& -\left(\alpha_{i 1}^{*}-\alpha_{i 2}\right) Q_{i} \bar{P}_{i} e^{-\left(\alpha_{i 2}+\alpha_{i 1}^{*}\right) x}+\left(\alpha_{i 2}^{*}-\alpha_{i 1}\right) \bar{Q}_{i} P_{i} e^{\left.-\alpha_{i 1}+\alpha_{i 2}^{*}\right) x},
\end{aligned}
$$

Using the assumption $\lambda_{1}^{*}<\lambda_{2}^{*}<\lambda_{2}<\lambda_{1}$ and the fact the $\lambda_{i}^{*}+(n-1) \lambda_{i}=1, i=1,2$, it follows, $\alpha_{i 1}+\alpha_{i 2}^{*}<\alpha_{i 1}+\alpha_{i 2}, \alpha_{i 1}+\alpha_{i 2}^{*}>\alpha_{i 1}^{*}+\alpha_{i 2}^{*}, \alpha_{i 1}+\alpha_{i 2}^{*}>\alpha_{i 1}^{*}+\alpha_{i 2}$ and all $\left(\alpha_{i 1}-\alpha_{i 2}\right)$, $\left(\alpha_{i 2}^{*}-\alpha_{i 1}^{*}\right),\left(\alpha_{i 2}-\alpha_{i 1}^{*}\right)$, are nonnegative. Using these observations in (5.13), we see

$$
g(x) \geq e^{-\left(\alpha_{i 1}+\alpha_{i 2}^{*}\right) x}\left\{-\left(\alpha_{i 1}-\alpha_{i 2}\right) P_{i} Q_{i}+\left(\alpha_{i 2}^{*}-\alpha_{i 1}^{*}\right) \bar{P}_{i} \bar{Q}_{i}\right.
$$




$$
\begin{aligned}
& \left.-\left(\alpha_{i 1}-\alpha_{i 2}^{*}\right) \bar{Q}_{i} P_{i}+\left(\alpha_{i 2}-\alpha_{i 1}^{*}\right) Q_{i} \bar{P}_{i}\right\} \\
= & \frac{e^{-\left(\alpha_{i 1}+\alpha_{i 2}^{*}\right) x}}{n-1}\left\{Q_{i}-P_{i}-\left(n Q_{i}-(i-1)\right) \lambda_{2}^{*}+\left(n P_{i}-(i-1)\right) \lambda_{1}^{*}\right\} \\
\geq & \frac{e^{-\left(\alpha_{i 1}+\alpha_{i 2}^{*}\right) x}}{n-1}\left\{Q_{i}-P_{i}-n\left(Q_{i}-P_{i}\right) \lambda_{2}^{*}\right\} \\
= & \frac{e^{-\left(\alpha_{i 1}+\alpha_{i 2}^{*}\right) x}}{n-1}\left(Q_{i}-P_{i}\right)\left(1-n \lambda_{2}^{*}\right) \\
\geq & 0 .
\end{aligned}
$$

The inequality in (5.14) follows, since by Lemma $5.1 P_{i} \leq \frac{i-1}{n}$ and $\lambda_{1}^{*}<\lambda_{2}^{*}$. From Lemma 5.2 it follows that $Q_{i} \geq P_{i}$, since it is known the likelihood ratio ordering implies usual stochastic ordering. This observation along with the fact that $\lambda_{2}^{*} \leq 1 / n$ implies the inequality in (5.15).

Remark : The conclusion of Theorem 5.2 holds if instead of $\lambda_{1}^{*}<\lambda_{1}$ and $\lambda_{2}^{*}<\lambda_{2}$ we assume that $\lambda_{1}^{*}>\lambda_{1}$ and $\lambda_{2}^{*}>\lambda_{2}$.

It is known that spacings of independent exponential random variables have $D F R$ distributions (cf. Kochar and Korwar, 1996). Combining this observation with Theorem 2.1, we have proved the following corollary.

Corollary 5.1 Under the assumptions of Theorem 5.2,

$$
D_{i: n}^{(1)} \geq_{\text {disp }} D_{i: n}^{(2)}
$$

A consequence of Corollary 5.1 is that $\operatorname{var}\left(D_{i: n}^{(1)}\right) \geq \operatorname{var}\left(D_{i: n}^{(2)}\right), i=1, \ldots, n$.

\section{Stochastic ordering for sample range}

Sample range is one of the criteria for comparing variabilities among distributions and hence it is important to study its stochastic properties. First we study the stochastic properties of the range of a random sample from a continuous distribution. Let $X_{1}, \ldots, X_{n}$ be a random sample from $F$ and let $Y_{1}, \ldots, Y_{n}$ be an independent random sample from another distribution $G$. It follows from Lemma 3(c) of Bartoszewic (1986) that $X \geq_{\text {disp }} Y \Rightarrow X_{n: n}-X_{1: n} \geq_{s t} Y_{n: n}-Y_{1: n}$. This observation along with Theorem 2.1 (a) leads to the following theorem.

Theorem 6.1 Let $X \geq_{h r} Y$ and let either $F$ or $G$ be DFR. Then

$$
X_{n: n}-X_{1: n} \geq_{s t} Y_{n: n}-Y_{1: n} .
$$

Next we consider the case when the parent observations are independent exponentials but with unequal parameters. Let $X_{1}, \ldots, X_{n}$ be independent exponential random variables with $X_{i}$ having hazard rate $\lambda_{i}, i=1, \ldots, n$. Let $Y_{1}, \ldots, Y_{n}$ be a random sample of size $n$ from an exponential distribution with common hazard rate $\bar{\lambda}$, the arithmetic mean of the $\lambda_{i}$ 's. Finally, let $R_{X}=X_{n: n}-X_{1: n}$ and $R_{Y}=Y_{n: n}-Y_{1: n}$ denote the sample ranges of $X_{i}$ 's and $Y_{i}$ 's, respectively. Kochar and Rojo (1996) proved that $R_{X} \geq_{s t} R_{Y}$. Khaledi and Kochar (2000 c) proved the following result which is in terms of $\tilde{\lambda}$, the geometric mean of the $\lambda_{i}$ 's. 
Theorem 6.2 Let $X_{1}, \ldots, X_{n}$ be independent exponential random variables with $X_{i}$ having hazard rate $\lambda_{i}$, for $i=1, \ldots, n$. Let $Y_{1}, \ldots, Y_{n}$ be a random sample of size $n$ from an exponential distribution with common hazard rate $\tilde{\lambda}$. Then,

$$
R_{X} \geq_{s t} R_{Y}
$$

Proof : The distribution function of $R_{X}$ (see David, 1981, p. 26) is

$$
F_{R_{X}}(x)=\frac{1}{\sum_{i=1}^{n} \lambda_{i}} \sum_{i=1}^{n} \frac{\lambda_{i}}{1-e^{-\lambda_{i} x}} \prod_{i=1}^{n}\left(1-e^{-\lambda_{i} x}\right) .
$$

and that of $R_{Y}$ is

$$
G_{R_{Y}}(x)=\left(1-e^{-\tilde{\lambda} x)}\right)^{n-1}
$$

Using (6.2) and (6.3), we have to show that

$$
\sum_{i=1}^{n} \frac{\lambda_{i}}{1-e^{-\lambda_{i} x}} \prod_{i=1}^{n}\left(1-e^{-\lambda_{i} x}\right) \leq \sum_{i=1}^{n} \lambda_{i}\left(1-e^{-\tilde{\lambda} x)}\right)^{n-1} .
$$

Multiplying both sides of (6.4) by $x(>0)$, it is sufficient to prove that

$$
\sum_{i=1}^{n} \frac{\lambda_{i} x}{1-e^{-\lambda_{i} x}} \prod_{i=1}^{n}\left(1-e^{-\lambda_{i} x}\right) \leq\left(\sum_{i=1}^{n} \lambda_{i} x\right)\left(1-e^{-\tilde{\lambda} x)}\right)^{n-1} .
$$

Dykstra, Kochar and Rojo (1997) proved that

$$
\sum_{i=1}^{n} \frac{y_{i}}{1-e^{-y_{i}}} \leq\left(\sum_{i=1}^{n} y_{i}\right) \prod_{i=1}^{n}\left(1-e^{-y_{i}}\right)^{-\frac{1}{n}}
$$

where $y_{i}>0$ for $i=1, \ldots, n$. Making use of this inequality on the L.H.S. of (6.5), we get

$$
\sum_{i=1}^{n} \frac{\lambda_{i} x}{1-e^{-\lambda_{i} x}} \prod_{i=1}^{n}\left(1-e^{-\lambda_{i} x}\right) \leq\left(\sum_{i=1}^{n} \lambda_{i} x\right) \prod_{i=1}^{n}\left(1-e^{-\lambda_{i} x}\right)^{\frac{n-1}{n}}
$$

A consequence of Theorem $4.4(b)$ is that $X_{n: n} \geq_{s t} Y_{n: n}$, which is equivalent to $\prod_{i=1}^{n}(1-$ $\left.e^{-\lambda_{i} x}\right)^{1 / n} \leq 1-e^{-\tilde{\lambda} x}$. Using this result, we find that the expression on the R.H.S. of (6.6) is less than or equal to that on the R.H.S. of (6.5) and from which the required result follows.

As a consequence of this result we get the following upper bound on the distribution function of $R_{X}$ in terms $\tilde{\lambda}$.

Corollary 6.1 Under the conditions of Theorem 6.2, for $x>0$,

$$
P\left[X_{n: n}-X_{1: n} \leq x\right] \leq\left[1-e^{-\tilde{\lambda} x}\right]^{n-1} .
$$

This bound is better than the one obtained in Kochar and Rojo (1996) in terms of $\bar{\lambda}$, since the expression on the R.H.S. of (6.7) is increasing in $\tilde{\lambda}$ and $\tilde{\lambda} \leq \bar{\lambda}$.

Now we extend Theorem 6.1 to the PHR model. We assume that $F$ is new worse than used (NWU), that is,

$$
\bar{F}(x+y) \geq \bar{F}(x) \bar{F}(y), \quad \text { for } x, y \geq 0,
$$


or equivalently,

$$
H(x+y) \leq H(x)+H(y), \quad \text { for } x, y \geq 0,
$$

where $H(x)=-\log \bar{F}(x)$ denotes the cumulative hazard of $F$.

Theorem 6.3 Let $X_{1}, \ldots, X_{n}$ be independent random variables with $X_{i}$ having survival function $\bar{F}^{\lambda_{i}}(x), \quad i=1, \ldots, n$. Let $Y_{1}, \ldots, Y_{n}$ be a random sample of size $n$ from a distribution with survival function $\bar{F}^{\tilde{\lambda}}(x)$, where $\tilde{\lambda}=\left(\prod_{i=1}^{n} \lambda_{i}\right)^{1 / n}$. If $F$ is $N W U$, then $X_{n: n}-X_{1: n} \geq_{s t} Y_{n: n}-Y_{1: n}$.

PROOF :

The distribution function of the sample range $X_{n: n}-X_{1: n}$ (see David, 1981, p. 26) is

$$
\begin{aligned}
F_{R_{n}^{X}}(x) & =\sum_{i=1}^{n} \int_{0}^{+\infty} \lambda_{i} h(t) e^{-\lambda_{i} H(t)} \prod_{j \neq i}^{n}\left(e^{-\lambda_{j} H(t)}-e^{-\lambda_{j} H(t+x)}\right) d t \\
& \leq \sum_{i=1}^{n} \int_{0}^{+\infty} \lambda_{i} h(t) e^{-\lambda_{i} H(t)} \prod_{j \neq i}^{n}\left(e^{-\lambda_{j} H(t)}-e^{-\lambda_{j} H(t)} e^{-\lambda_{j} H(x)}\right) d t
\end{aligned}
$$

(since $F$ is NWU )

$$
\begin{aligned}
& =\sum_{i=1}^{n} \lambda_{i} \prod_{j \neq i}\left(1-e^{-\lambda_{j} H(x)}\right) \int_{0}^{+\infty} h(t) \prod_{j=1}^{n} e^{-\lambda_{j} H(t)} d t \\
& =\sum_{i=1}^{n} \lambda_{i} \prod_{j \neq i}\left(1-e^{-\lambda_{j} H(x)}\right) \int_{0}^{+\infty} h(t) e^{-H(t) \sum_{j=1}^{n} \lambda_{j}} \\
& =\frac{1}{\sum_{i=1}^{n} \lambda_{i}} \sum_{i=1}^{n} \frac{\lambda_{i}}{1-e^{-\lambda_{i} H(x)}} \prod_{i=1}^{n}\left(1-e^{-\lambda_{i} H(x)}\right), \quad x>0,
\end{aligned}
$$

Now, replacing $x$ with $H(x)$ in the proof of Theorem 6.2, it is easy to see that

$$
F_{R_{n}^{X}}(x) \leq F_{R_{n}^{Y}}(x)
$$

\section{References}

1. Arnold, B. C., Balakrishnan, N., and Nagaraja, H. N. (1992). A First Course in Order Statistics. Wiley, New York.

2. Bagai, I. and Kochar, S. C. (1986). On tail ordering and comparison of failure rates. Comm. Statist. Theory and Methods 15, 1377-1388.

3. Balakrishnan, N. and Rao, C. R. (1998a). Handbook of Statistics 16 - Order Statistics : Theory and Methods. Elsevier, New York.

4. Balakrishnan, N. and Rao, C. R. (1998b). Handbook of Statistics 17 - Order Statistics : Applications. Elsevier, New York.

5. Bapat, R. B. and Beg, M. I. (1989). Order statistics for nonidentically distributed variables and permanents. Sankhya Ser. B 51, 79-93. 
6. Bapat, R. B. and Kochar, S. C. (1994). On likelihood ratio ordering of order statistics. Linear Algebra and Its Applications 199, 281-291.

7. Barlow, R. E. and Proschan, F. (1966). Inequalities for linear combinations of order statistics from restricted families. Ann. Math. Statist. 37, 1574-1592.

8. Barlow, R. E. and Proschan, F. (1981). Statistical Theory of Reliability and Life Testing. To Begin With : Silver Spring, Maryland.

9. Bartoszewicz, J. (1986). Dispersive ordering and the total time on test transformation. Statist. Probab. Lett. 4, 285- 288.

10. Bartoszewicz, J. (1987). A note on dispersive ordering defined by hazard functions. Statist. Probab. Lett. 6, 13-17.

11. Boland, P.J., El-Neweihi, E. and Proschan, F. (1994). Applications of the hazard rate ordering in reliability and order statistics. J. Appl. Probab. 31, 180-192.

12. Boland, P. J., Hollander, M., Joag-Dev, K. and Kochar, S. (1996). Bivariate dependence properties of order statistics. J. Multivariate Anal. 56, 75-89.

13. Boland, P.J., Shaked, M. and Shanthikumar, J.G. (1998). Stochastic ordering of order statistics. In N. Balakrishnan and C. R. Rao, eds, Handbook of Statistics 16 - Order Statistics : Theory and Methods. Elsevier, New York, 89-103. [Technical report of the University College Dublin, 1995].

14. Bon, J. L. and Paltanea, E. (1999). Ordering properties of convolutions of exponential random variables. Lifetime Data Anal. 5, 185- 192.

15. Chan, W., Proschan, F. and Sethuraman, J. (1991). Convex ordering among functions, with applications to reliability and mathematical statistics. In Topics in Statistical Dependence, ed. H. W. Block, A. R. Sampson and T. H. Savits. IMS Lecture Notes 16, 121-134.

16. David, H. A. (1981). Order Statistics (2nd ed.). Wiley, New York.

17. David, H.A. and Groeneveld, R.A. (1982). Measures of local variation in a distribution: Expected length of spacings and variances of order statistics. Biometrika 69, 227-232.

18. Deshpande, J. V. and Kochar, S. C. (1983). Dispersive ordering is the same as tail ordering. Adv. in Appl. Probab. 15, 686-687.

19. Dharmadhikari, S. and Joeg-dev, K. (1988). Unimodality, Convexity and Applications. Academic press, INC.

20. Dykstra, R., Kochar, S. C. and Rojo, J. (1997). Stochastic comparisons of parallel systems of heterogeneous exponential components. J. Statist. Plann. Inference 65, 203-211.

21. Khaledi, B. and Kochar, S. C. (1999 ). Stochastic ordering between distributions and their sample spacings. Statist. Probab. Lett. 44, 161-166.

22. Khaledi, B. and Kochar, S. C. (2000 a). On dispersive ordering among order statistics in one-sample and two-sample problems. Statist. Probab. Lett. 46, 257-261. 
23. Khaledi, B. and Kochar, S. C. (2000 b). Some new results on stochastic comparisons of parallel systems. J. Appl. Probab. 37,1123-1128.

24. Khaledi, B. and Kochar, S. C. (2000 c). Sample range-some stochastic comparisons results. Calcutta Statistical Association Bulletin 50, 283-291.

25. Khaledi, B. and Kochar, S. C. (2001). Stochastic properties of Spacings in a SingleOutlier Exponential Model. Probability in Engineering and Information Sciences 15 (2001), 401-408

26. Kochar, S. C. (1996). Dispersive ordering of order statistics. Statist. Probab. Lett. 27, 271-274.

27. Kochar, S. C. (1998). Stochastic comparisons of spacings and order statistics. Frontiers in Reliability. World Scientific : Singapore. 201-216. eds., Basu, A. P., Basu, S. K. and Mukhopadhyay, S.

28. Kochar, S.C. (1999). On stochastic ordering between distributions and their sample spacings. Statist. Probab. Lett. 42, 345-352.

29. Kochar, S. C. and Kirmani, S.N.U.A. (1995). Some results on normalized spacings from restricted families of distributions. J. Statist. Plann. Inference 46, 47-57.

30. Kochar, S. C. and Korwar, R. (1996). Stochastic orders for spacings of heterogeneous exponential random variables. J. Multivariate Anal. 57, 69-83.

31. Kochar, S. C. and Ma, C. (1999). Dispersive ordering of convolutions of exponential random variables. Statist. Probab. Lett. 43, 321-324.

32. Kochar, S. C. and Rojo, J. (1996). Some new results on stochastic comparisons of spacings from heterogeneous exponential distributions. J. Multivariate Anal. 59, 272-281.

33. Lewis, T. and Thompson, J. W. (1981). Dispersive distribution and the connection between dispersivity and strong unimodality. J. Appl. Probab. 18, 76-90.

34. Lillo, R. E., Nanda, A.K. and Shaked, M (2000) Preservation of some likelihood ratio stochastic orders by order statistics. Statistics and Probability Letters, To appear.

35. Lynch, J. Mimmack, G. and Proschan, F. (1987). Uniform stochastic orderings and total positivity. Canad. J. Statist. 15,63-69.

36. Marshall, A. W. and Olkin, I. (1979). Inequalities : Theory of Majorization and Its Applications. Academic Press, New York.

37. Mitrinovic, D. S. (1970). Analytic Inequalities. Springer Verlag, Berlin.

38. Nappo, G. and Spizzichino, F. (1998). Ordering properties of the TTT-plot of lifetimes with Schur joint densities. Statist. Probab. Lett. 39, 195-203.

39. Pledger, P. and Proschan, F. (1971). Comparisons of order statistics and of spacings from heterogeneous distributions. Optimizing Methods in Statistics. Academic Press, New York., 89-113. ed. Rustagi, J. S. 
40. Proschan, F. and Sethuraman, J. (1976). Stochastic comparisons of order statistics from heterogeneous populations, with applications in reliability. J. Multivariate Anal. 6, 608616 .

41. Raqab, M.Z. and Amin, W.A. (1996). Some ordering results on order statistics and record values. IAPQR Transactions. 21, No. 1, 1-8.

42. Rojo, J. and He, G.Z. (1991). New properties and characterizations of the dispersive ordering. Statist. Probab. Lett. 11, 365-372.

43. Ross, S.M. (1983). Stochastic Processes. Wiley, New York.

44. Saunders, D.J. (1984). Dispersive ordering of distributions. Adv. Appl. Prob. 16, 693-694

45. Saunders, I. W. and Moran, P. A. P. (1978). On quantiles of the gamma and $F$ distributions. J. Appl. Probab. 15, 426-432.

46. Sen, P. K. (1970). A note on order statistics for heterogeneous distributions. Ann. Math. Statist. 41, 2137-2134.

47. Shaked, M. and Shanthikumar, J. G. (1994). Stochastic Orders and their Applications. Academic Press, San Diego, CA.

48. Shaked, M. and Tong, Y. L. (1984). Stochastic ordering of spacings from dependent random variables. Inequalities in Statistics and Probability, IMS Lecture Notes-Monograph series, 5, 141-149. 